\title{
Omnidirectional Band Gaps in Heterostructure Materials Composed of Meta-materials and Magnetic Materials
}

\author{
J P Pandey ${ }^{1}$, G N Pandey ${ }^{2}$ \\ ${ }^{1}$ Department of Physics, M. L. K. P. G. College, Balrampur (UP), India. \\ ${ }^{2}$ Department of Physics, Amity Institute of Applied Sciences, Amity University, Noida (U.P.)
}

\begin{abstract}
:
Enlarged band gap in photonic heterostructure (PC1/PC2) composed of metamaterials and magnetic materials is studied using simple transfer matrix method. As we know that the meta-materials have the unusual electromagnetic properties having negative refractive index. The negative index material is affected with their optical parameters like refractive index, permittivity, permeability and thickness of the materials. The PC1 and PC2 are two photonic crystals containing NIM with different thickness ratio. When these two PCs are combined and make a heterostructure of PC1/PC2 of different thicknesses ratio, then the band gap of such heterostructure is enlarged due to the band gap of PC1 and PC2. Such structure may be used as a broad band filter for TE and TM modes.
\end{abstract}

\section{INTRODUCTION:}

The heterostructure consists of two 1-D periodic photonic structures with different photonic stop bands. Multiple heterostructures are formed if several 1-D PCs with different layer thicknesses are combined. We found that the band gap of the heterostructures was extended obviously. This enlargement can be done in different ways: (i) by stacking together two or more different multilayers, i.e. by using photonic heterostructures, $[1,2]$ (ii) by increasing the refractive index contrast [3]; (iii) by using the chirped structure, where the thickness of the period is increased continuously instead of being constant [4]; (iv) by using the disordered structures [5]; (v) by introducing defect in periodic structures [6]. Heterostructure engineering is now widely practiced, producing the most efficient semiconductor lasers, highest-speed transistors, and novel quantum electronic devices.

If the nontransmission frequency range (PBG along the incident direction) of the constituents has a proper lineup, this can be essentially enlarged as desired by using different PCs to form photonic multiple heterostructures. The directional PBGs for different PCs overlap each other at any incident angle. This kind of lineup of PBGs is crucial for the enlargement of the omnidirectional total reflection by using two or more PCs to form photonic heterostructures.

The negative refractive index of the material is caused by negative value of the dielectric permittivity and magnetic permeability for certain frequency. For a wave propagating in the material, the electric field vector and wave vector form the left set of vectors. That is why the negative refractive index media are often called left handed materials (NIM) or metamaterials.

The angular dependence of the band gap for the 1-D PCs consisting of alternating slabs made of PIM and NIM are studied by Daninthe et al. [7]. They found an omni gap, around the frequency where $\mathrm{n}_{\mathrm{av}}$ is zero, for a certain type of constituent lefthanded medium which is independent of both angle and polarization. G N Pandey et al. theoretically studied omni-directional reflection band in one dimensional plasma photonic crystal in TE- and TMmodes. They also studied the reflectance and transmittance behaviors of superconductor dielectric photonic crystal at different incidence angles and temperature [8-11]. J P Pandey also studied about the enlargement of omnidirectional bandgaps in various PCs [12-13].

- In this paper, we have studied the optical properties of the periodic structure with filter characteristics containing PIMs and NIMs. The electromagnetic wave propagation in periodic structure is studied by calculating the plane wave equation in each medium. To study the wave characteristics, we have to know the Bloch wave and the transfer matrix method. In next section, we will study the Bloch's wave and TMM method. 


\section{THEORY AND METHODOLOGY:}

For an infinite grating extending on the whole z-axis, the solution of the Helmholtz equation can be written in term of Bloch waves,

$$
E(z)=u(z) e^{i K(\omega) z}
$$

where $\mathrm{u}(\mathrm{z})$ is a complex valued periodic function with the period of the grating, $\mathrm{u}(\mathrm{z})=\mathrm{u}(\mathrm{z}+\mathrm{d})$. The parameter $K(\omega)$ is called the Bloch wave number and

$$
K(\omega)=\frac{1}{d} \cos ^{-1}\left(\frac{1}{2} \operatorname{Trace}[M]\right)
$$

where the symbols have their usual meaning.

\section{Transfer Matrix Techniques for 1-D Photonic} crystals:-

The simplest way to describe the wave behavior in 1-D grating is by using the transfer matrix method (TMM) [14]. The transfer matrix of
(1)

for a periodic grating with indices $\mathrm{n}_{1}$ and $\mathrm{n}_{2}$, there is an explicit expression for $\mathrm{k}(\omega)$ as follows;

one unit grating will be derived below. Consider a periodic arrangement of multilayer films, with refractive indices $n_{1}$ and $n_{2}$ and thicknesses $d_{1}$ and $d_{2}$ respectively. The solution will be the superposition of plane waves traveling to the right and to the left.

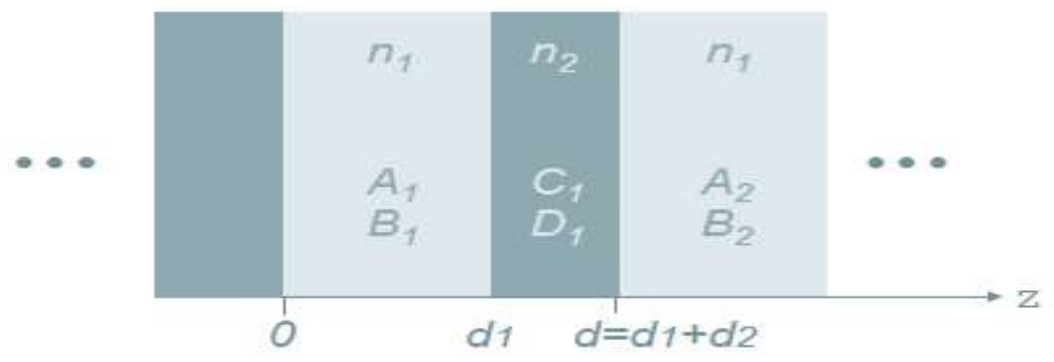

Figure1: Periodic arrangement of multilayer media with refractive indices $n_{1}$ and $n_{2}$.

Say, for layer with index $\mathrm{n}_{1}$ the right going and left going plane waves amplitudes are $\mathrm{A}_{1}$ and $\mathrm{B}_{1}$ respectively and for layer with index $n_{2}$, the right

$$
E(z)=A_{1} e^{i k_{1} z}+B_{1} e^{-i k_{1} z}
$$

going plane waves have amplitudes $\mathrm{C}_{1}$ and $\mathrm{D}_{1}$ respectively. Hence for layer with index $\mathrm{n}_{1}$ the solution

and

$$
E(z)=C_{1} e^{i k_{2}\left(z-d_{1}\right)}+D_{1} e^{-i k_{2}\left(z-d_{1}\right)}
$$

for the layer with index $n_{2}$, the parameters $k_{1}$ and $k_{2}$ will be called the wave numbers. At the interface between layers $\left(z=d_{1}\right)$, the solution and its derivative should be continuous. This gives a relation between plane wave's amplitudes, $\left[\begin{array}{l}C_{1} \\ D_{1}\end{array}\right]=M_{12}\left[\begin{array}{l}A_{1} \\ B_{1}\end{array}\right]$

With

$$
\left\{\begin{array}{l}
\frac{1}{2}\left(1+\frac{k_{1}}{k_{2}}\right) e^{i k_{1} d_{1}} \frac{1}{2}\left(1-\frac{k_{1}}{k_{2}}\right) e^{-i k_{1} d_{1}} \mid \\
\frac{1}{2}\left|1-\frac{k_{1}}{k_{2}}\right| e^{i k_{1} d_{1}} \frac{1}{2}\left|1+\frac{k_{1}}{k_{2}}\right| e^{-i k_{1} d_{1}} \mid
\end{array} \mid\right.
$$


and also at $\mathrm{z}=\mathrm{d}$, the interface between layers with index $n_{2}$ and $n_{1}$ continuity of the plane waves and its

derivative gives, $\left[\begin{array}{l}\left.A_{2}\right\rceil \\ B_{2}\end{array}\right]=M_{21}\left[\begin{array}{l}C_{1} \\ D_{1}\end{array}\right]$

where the matrix $M_{21}$ is the same as $M_{12}$ but with interchanging the indices. Concluding from the two matrix

equations above,

$$
\left\lceil\frac{A_{2}}{B_{2}}\right\rfloor=M\left\lceil\frac{A_{1}}{B_{1}}\right\rfloor
$$

where $M=M_{21} M_{12}$.

Matrix $M$ is called as the Transfer Matrix method of one unit of grating. Using the transfer Matrix technique, it can be shown easily that the relation

between the plane waves amplitudes in the left and right exterior is as follows;

$$
\left\lceil\begin{array}{l}
t\rceil \\
0
\end{array}\right\rfloor=M\left[\begin{array}{l}
1\rceil \\
r
\end{array}\right\rfloor
$$

where $t$ and $r$ is the transmittance and reflectance of the wave and the value of $\mathrm{M}$ is-

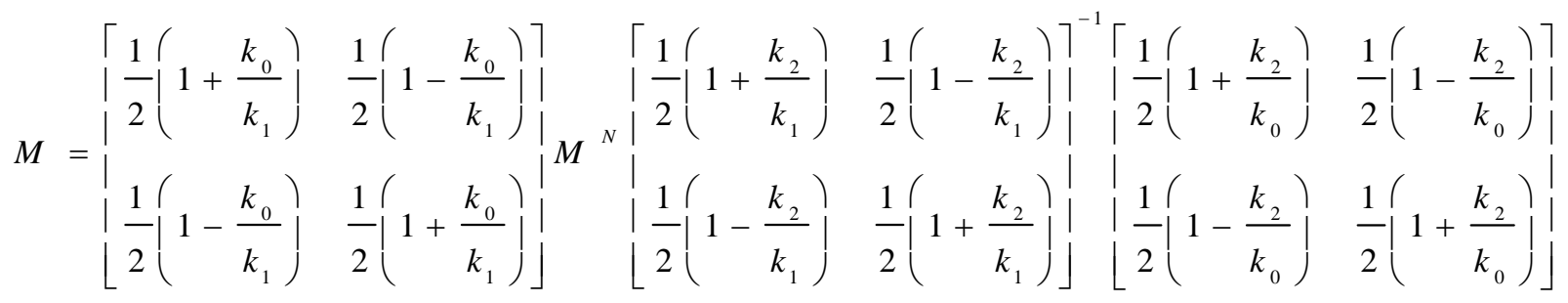

with $\mathrm{M}$ given earlier and the power transfer is given by a quantity called the transmittance, defined as;

$$
T=|t|^{2}
$$

Now if we plot a graph between normalized frequency vs transmittance, then it gives the complete photonic band gap.

Physical model and calculation method for heterostructure containing negative index materials-

1-D PCs composed of alternating layers of negative index materials (NIM) and positive index materials (PIM) are shown in figure 2. 1D-PCs are
PC1 $(A B)^{m}$ and PC2 $\left(A B_{1}\right)^{n}$ where $\mathrm{A}$ and $B_{1}$ are NIM and PIM respectively, $\mathrm{m}(\mathrm{n})$ is the period number. Here we suppose that the relative permittivity and permeability in the NIMs are given by

$$
\begin{aligned}
\varepsilon_{A}=1.21-\left(\omega_{e p}\right)^{2} /\left(\omega^{2}+i \omega \gamma_{e}\right) \\
\mu_{A}=1-\left(\omega_{m p}\right)^{2} /\left(\omega^{2}+i \omega \gamma_{m}\right)
\end{aligned}
$$




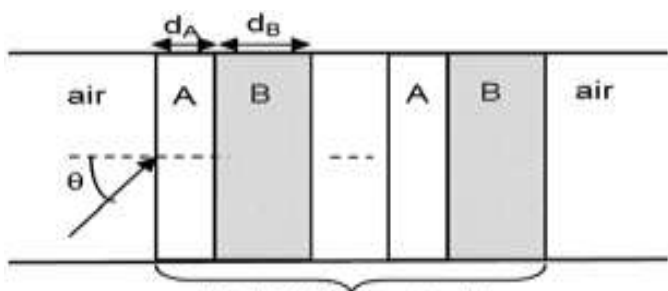

(a) $\mathrm{PC} 1$ ( $m$ periods)

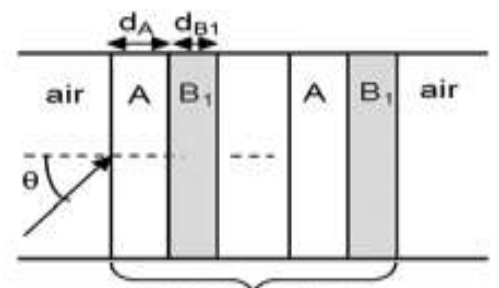

(b) PC2(n periods)
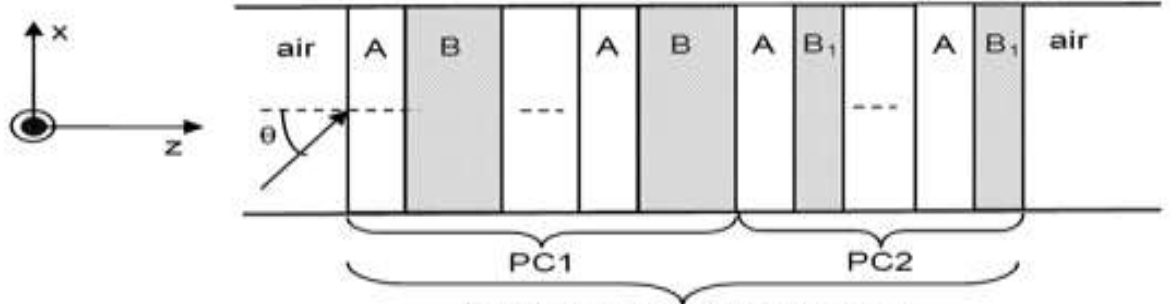

(c) Heterostructure[PC1PC2]

Figure 2: schematic diagrams of the (a) PC1, (b) PC2 and (c) PC1/PC2.

where $\omega_{e p}$ and $\omega_{m p}$ is the electronic and magnetic plasma frequencies and $\gamma_{e}$ and $\gamma_{m}$ denote the electric and magnetic damping factors that contribute to the absorption and losses. In above calculation, lossless NIM were considered i.e. $\gamma_{e}=0$ and $\gamma_{m}=0$.then above equation takes the form $\varepsilon_{A}=1.21-\left(\omega_{e p}\right)^{2} /\left(\omega^{2}\right)$

and

$$
\mu_{A}=1-\left(\omega_{m p}\right)^{2} /\left(\omega^{2}\right)
$$

For permittivity and permeability to be negative simultaneously, the frequency of range lies $\omega<\omega_{e p}$ and $\omega<\omega_{m p}$ for electronic and magnetic respectively. We have choosen plasma frequencies $\omega_{e p}=\omega_{m p}=10 \mathrm{GHz}$, similar form has been taken theoretically [15-16] and experimentally [17-18] in the previous report. The thickness of the NIM and PIM are $d_{A}$ and $d_{B}$ respectively with the length of unit cell is $\mathrm{d}\left(=d_{A}+d_{B}\right)$. Here we have taken PC1
(PC2) with constant thickness of A material, $d_{A}=12 \mathrm{~mm} \quad(12 \mathrm{~mm})$ and $d_{B}=24 \mathrm{~mm} \quad(6 \mathrm{~mm})$ respectively with $n_{A} d_{A}+n_{B} d_{B}=0$ [19]. The number of periods in PC1 and PC2 are 20 each i.e. $n=m=20$.

Let a plane wave is incident from the vacuum to the $1 \mathrm{DPC}$ with the incident angle $\theta$ with respect to $+\mathrm{z}$ direction. In general, the electric and magnetic fields at any two positions $\mathrm{z}$ and $\mathrm{z}+\Delta \mathrm{z}$ in the same layer are connected via a transfer matrix which is discussed earlier [14]

$$
M_{j}(\Delta z, \omega)=\left(\begin{array}{cc}
\cos \left(k_{z}^{j} \Delta z\right) & i \frac{1}{q_{j}} \sin \left(k_{z}^{j} \Delta z\right) \\
i q_{j} \sin \left(k_{z}^{j} \Delta z\right) & \cos \left(k_{z}^{j} \Delta z\right)
\end{array}\right) \quad \text { where }
$$

$\left.k_{z}^{j}=\left(\frac{\omega}{c}\right) \sqrt{ } \epsilon_{j}\right) \sqrt{\mu_{j}} \sqrt{ }\left(1-\sin ^{2} \theta / \varepsilon_{j} \mu_{j}\right)$ is the $\mathrm{Z}$ component of the wave vector $k_{j}$ in the $\mathrm{j}$ 'th layer, $\mathrm{c}$ is the speed of light in the vacuum and $\left.q_{j}=\sqrt{\epsilon_{j}}\right) / \sqrt{ } \mu_{j} \sqrt{ }\left(1-\sin ^{2} \theta / \varepsilon_{j} \mu_{j}\right)$ is the component of the vector along the interface. The transmission coefficient $\mathrm{t}(\omega, \theta)$ can be expressed as

$$
t(\omega)=\frac{2 \cos \theta}{\cos \theta\left[x_{11}(\omega)+x_{22}(\omega)\right]+i\left[\cos 2 \theta x_{12}(\omega)-x_{21}(\omega)\right]}
$$

(Here $x_{i j} \quad(\mathrm{i}, \mathrm{j}=1,2)$ is the matrix element of $X_{N}(\omega)=\Pi_{j=1}^{N} M_{j}\left(d_{j}, \omega\right)$, which represents the total transfer matrix connecting way. For an infinite periodic structure, according to the fields at the entrance and the exit The transverse Bloch's theorem, the dispersion at any incident magnetic (TM) wave can be treated in a similar angle obeys the relation

$\cos \left[K_{z}\left(d_{A}+d_{B}\right)\right]=\cos \left(k_{z}^{A} d_{A}\right) \cos \left(k_{z}^{B} d_{B}\right)-\frac{1}{2}\left(\frac{q_{B}}{q_{A}}+\frac{q_{A}}{q_{B}}\right) \sin \left(k_{z}^{A} d_{A}\right) \sin \left(k_{z}^{B} d_{B}\right)$

where $K_{z}$ is the component of the Bloch wave vector, $d_{A}$ and $d_{B}$ are the thicknesses of layers $A$ and $\mathrm{B}$ respectively. The condition of $\cos \left[K_{z}\left(d_{A}+d_{B}\right)\right]>1$ corresponds to the band gap of 1DPC and is known as the Bragg condition. Using above equation, we have calculated the transmittance of PC1 and PC2 separately and then transmittance PC1/PC2 of heterostructure is calculated with varying angle of incidence. 


\section{RESULTS AND DISCUSSION}

In this paper, we have calculated the refractive index of NIM and the average refractive index of the NIM-PIM structure. Besides this we have also calculated the reflectance of the PC1, PC2 and $\mathrm{PC} 1 / \mathrm{PC} 2$ and tried to calculate the omnidirectional behavior of $\mathrm{PC} 1 / \mathrm{PC} 2$ heterostructure.

\section{Filter characteristics of photonic crystal:-}

In this paper, we have concentrated our study on filter characteristics of heterostructure photonic crystal containing PC1 and PC2. Such calculation has been made by Xiang et al. [20] for PIM as a dielectric material and Thapa et al. have calculated filter characteristics of the hetero structure containing NIM and magnetic material of PIM. We

and

$$
\begin{aligned}
& \varepsilon_{A}=a-\left(\omega_{e p}\right)^{2} /\left(\omega^{2}\right) \\
& \mu_{A}=b-\left(\omega_{m p}\right)^{2} /\left(\omega^{2}\right)
\end{aligned}
$$

where $\mathrm{a}$ and $\mathrm{b}$ are positive constants, and $\omega_{e p}$ and $\omega_{m p}$ are the electronic and magnetic plasma frequency respectively. In the frequency range of have also studied the filter characteristics of heterostructure $\mathrm{PC} 1$ and $\mathrm{PC} 2$ containing NIM and PIM for getting large band gap by combining the PC1 and $\mathrm{PC} 2$ periodic structure.

Figures 3.1(a) and 3.2(a) have shown the refractive index of the NIM for PC1 and PC2 respectively. Now we have made the repetition of the PIM and NIM with different thickness ratio of the PIM and NIM. The average refractive index of the structure for $\mathrm{PC} 1$ and $\mathrm{PC} 2$ are shown in the figures 3.1(b) and 3.2(b) respectively. The reflectance spectrum of PC1 and PC2 having NIMs and PIMs with different thickness ratio are shown in the figures 3.1(c) and 3.2(c) respectively. Here we suppose that the relative permittivity and permeability in the NIMs are given by $\omega^{2}<\left(\omega_{e p}^{2} / \mathrm{a}, \omega_{m p}^{2} / \mathrm{b}\right), \varepsilon_{A}$ and $\mu_{A}$ are negative simultaneously. In the following calculation, we choose $\mathrm{a}=1.21, \mathrm{~b}=1$, and $\omega_{e p}=\omega_{m p}=10 \mathrm{GHz}$.

NIM
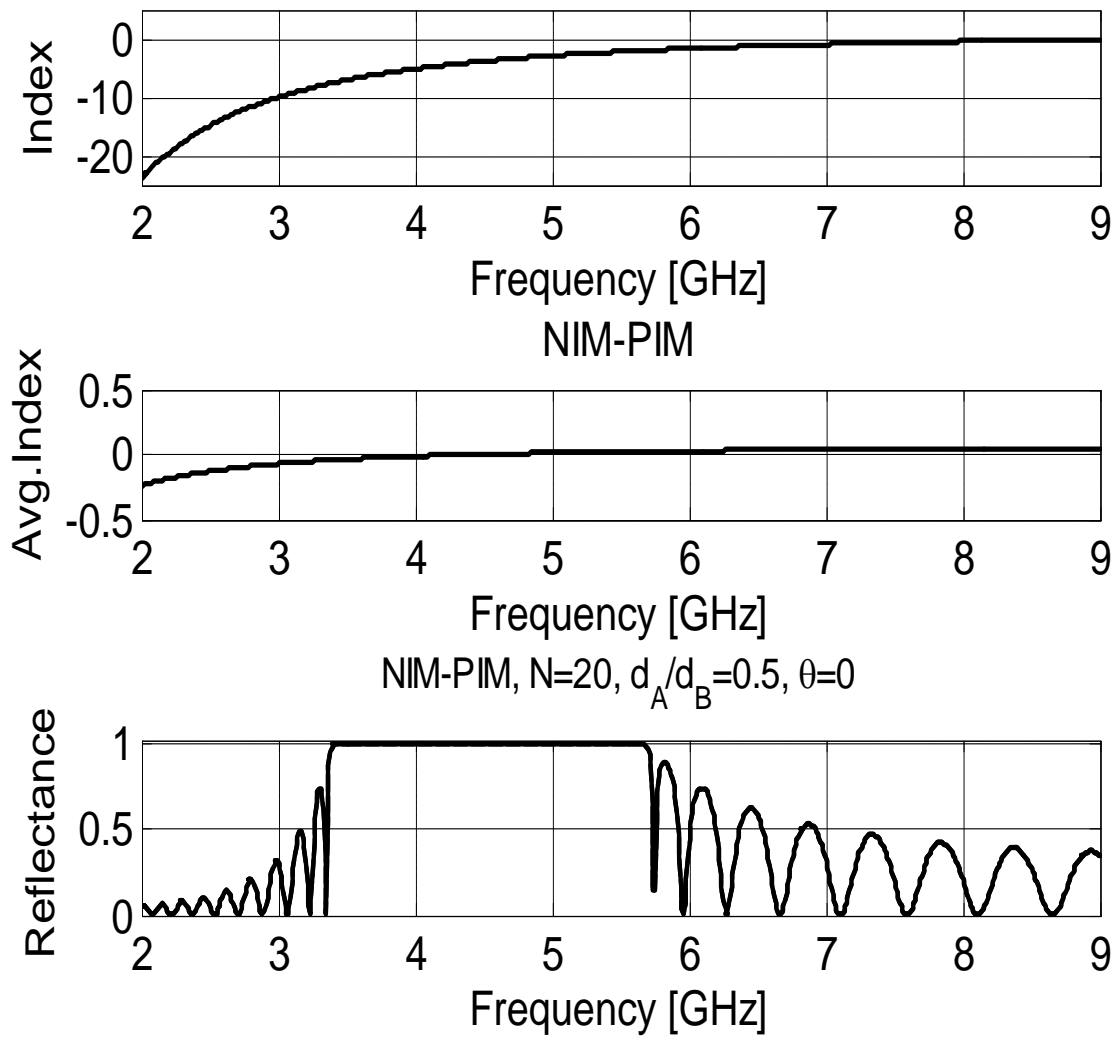

Figure 3.1: (a) Refractive index of NIM (b) Average refractive index of PIM-NIM and (c) Reflectance spectrum of PC1 having NIM-PIM with $\mathrm{d}_{\mathrm{A}} / \mathrm{d}_{\mathrm{B}}=0.5$ at normal incidence. 
The thickness of NIM and PIM in two 1DPCs are assumed to be $d_{A}$ and $d_{B}$ respectively. Here we assume $d_{A}=12 \mathrm{~mm}$ and $d_{B}=24 \mathrm{~mm}, \varepsilon_{B}=1$, $\mu_{B}=1$ and $\mathrm{m}=\mathrm{n}=20$. Under these conditions, when $n_{A} d_{A}+d_{B} n_{B}=0$, the effective zero refractive index condition $\left[\ddot{n}=(1 / \mathrm{d}) \int_{0}^{d} n(z) d z=0\right]$ will be satisfied.

Figures 3.1 and 3.2 represent the three parts of the optical parameters like: (1) refractive index of NIM, (2) average refractive index of NIM and PIM, and (3) optical reflectance for the considered structure versus frequency $(\mathrm{GHz})$ for thickness ratio $d_{A} / d_{B}=0.5$ for $\mathrm{PC} 1$ and $d_{A} / d_{B}=2.0$ for $\mathrm{PC} 2$ respectively when the thickness of NIM is $12 \mathrm{~mm}$. The band gap of the PC1 having NIM-PIM for normal incidence for TE Mode with thickness ratio $d_{A} / d_{B}=0.5$ is found $\Delta \omega=2.3 \mathrm{GHz}$ which is the totally matched with the zero refractive index of NIM and PIM as shown in figure 3.1. However the band

gap for TM mode of the same structure i.e. PC1 is also found same band gap.

The figures 3.1(b) and 3.2(b) i.e. average refractive index is affected with the thickness ratio of the PIM and NIM which is obviously obtained in $\mathrm{PC} 1$ and PC2. The average refractive index of PC1 and $\mathrm{PC} 2$ is found zero for $4.0-6.2 \mathrm{GHz}$ and $5.8-9.0$ $\mathrm{GHz}$ respectively. Similarly the band gap of $\mathrm{PC} 2$ for normal incidence for TE mode with thickness ratio $d_{A} / d_{B}=2.0$ is found $\Delta \omega=2.4 \mathrm{GHz}$ which is also the totally matched with the zero refractive index of NIM and PIM as shown in figure 3.2. The dependence of the photonic band gap with angle of incidence with the thickness ratio $d_{A} / d_{B}=0.5$ and $d_{A} / d_{B}=2.0$ are depicted in the figures 3.3respectively for TE and TM modes. The frequency band gap shifts towards lower frequency when thickness ratio is $d_{A} / d_{B}=2.0$ i.e large thickness of the PIM. The frequency band gap remains constant at the lower frequency when the thickness ratio is $d_{A} / d_{B}=0.5$.

NIM

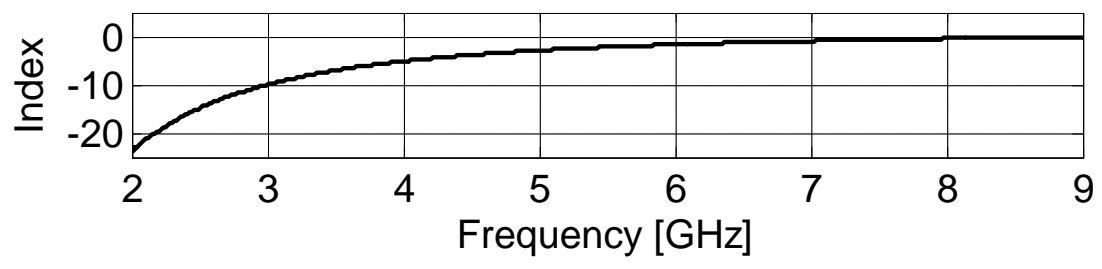

NIM-PIM

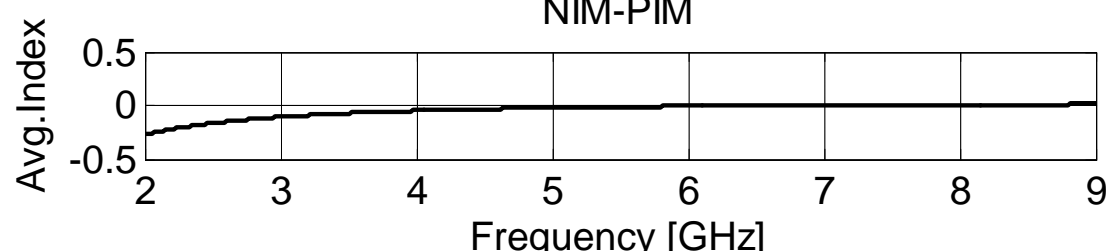

Frequency $[\mathrm{GHz}]$

NIM-PIM, $N=20, d_{A} / d_{B}=2, \theta=0$

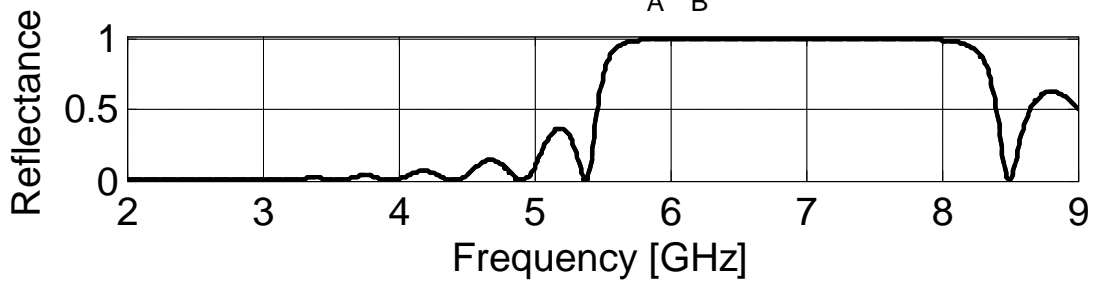

Figure 3.2: (a) Refractive index of NIM (b) Average refractive index of PIM-NIM and (c) Reflectance spectrum of PC2 having NIM-PIM with $\mathrm{d}_{\mathrm{A}} / \mathrm{d}_{\mathrm{B}}=2$ at normal incidence.

Now, we discuss the optical transmission spectra of PC1. Figure 3.3 gives the dependence of the PBGs on the incident angle for TE and TM modes which demonstrated the frequency band gap is insensitive to the incident angle for TM polarization but sensitive for TE polarization for the thickness ratio $d_{A} / d_{B}=0.5$. The photonic band gap has two frequencies i.e. first at the lower range and second at the higher frequency range. The frequency shift of the edges of frequency band gap is small when the incident angle increases for TM polarization. There is an omni-directional gap (OBG) for TE polarization in the frequency range, from $3.5-5.8 \mathrm{GHz}$ and for $\mathrm{TM}$ polarization, the OBG is observed from 3.6-5.8 GHz. 


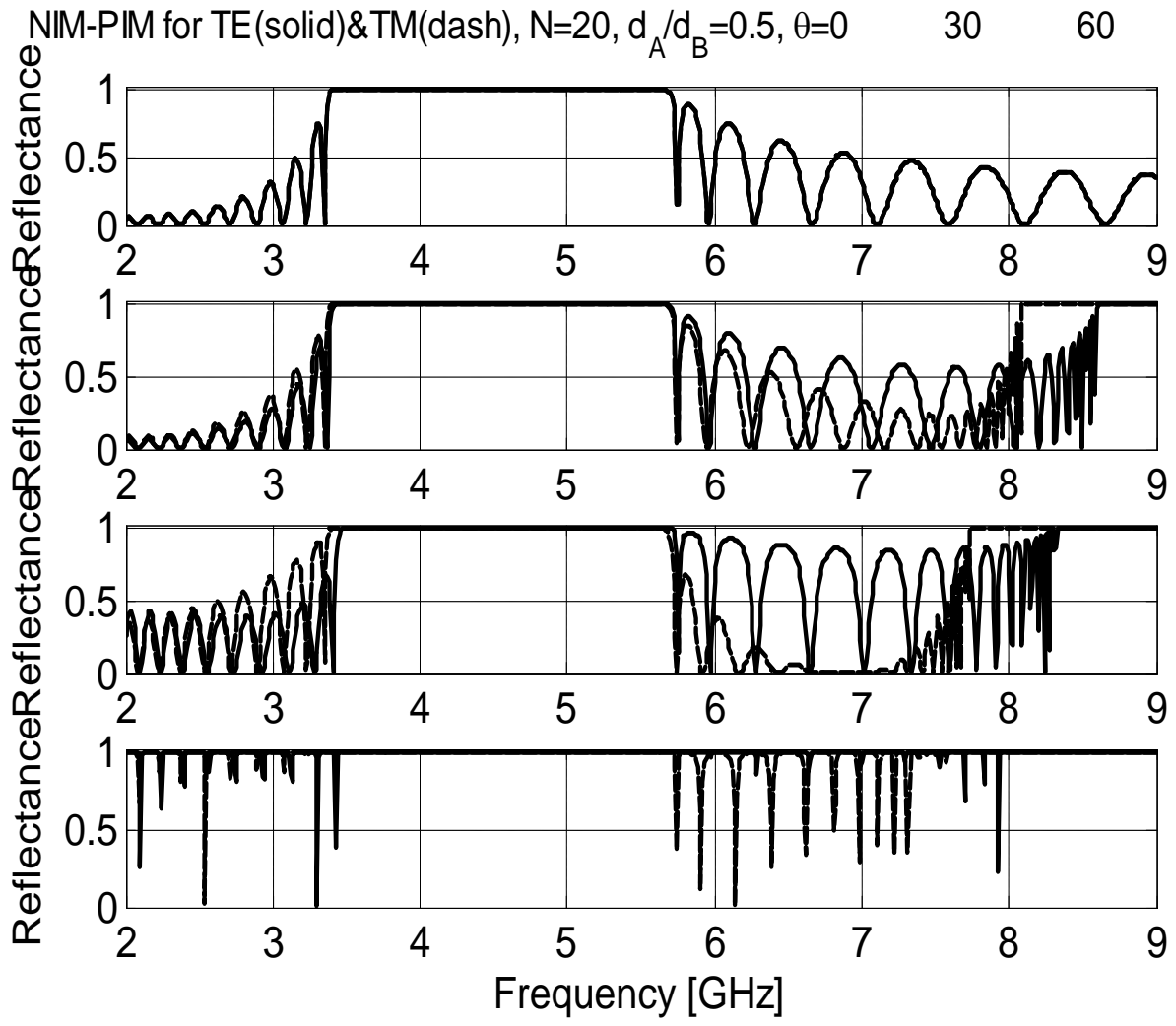

Figure 3.3: Reflectance spectrum of PC1 having NIM-PIM with $\mathrm{d}_{\mathrm{A}} / \mathrm{d}_{\mathrm{B}}=0.5$ at angle of incidence (a) $0^{\circ}$, (b) $30^{\circ}$, (c) $60^{\circ}$ and (d) $89^{\circ}$ respectively.

The overall frequency range of the OBG for any polarization is obtained from $3.5-5.8 \mathrm{GHz}$ and the frequency band width is $\Delta \omega=2.3 \mathrm{GHz}$, which is determined by the frequency range at the grazing incidence $\theta=90^{\circ}$ for both polarizations, viz, the OBGs in this 1DPC exist between the upper frequency edge at $\theta=90^{\circ}$ for TE polarization and the lower frequency edge polarization at $\theta=90^{\circ}$ for TM polarization.

\section{CONCLUSION:}

We have studied the physics of the negative index material (NIM) and concluded that the NIM is an artificial structure which is fabricated by the combination of the negative electric permittivity and negative magnetic permeability of the materials. We have made the physical modeling of the two photonic crystals on the basis of the thickness ratio of the PIM and NIM i.e. PC1 and PC2 with thickness ratios $\mathrm{d}_{\mathrm{A}} / \mathrm{d}_{\mathrm{B}}=0.5$ and $\mathrm{d}_{\mathrm{A}} / \mathrm{d}_{\mathrm{B}}=2$ respectively. By properly chosen the thickness ratio and average refractive index of the PIM and NIM, we have modulated the filter characteristics of the heterostructure $\mathrm{PC} 1$ and PC2. Using our theoretical approach for the heterostructure of PC1 and PC2, one can fabricate the enlarged band gap for omni-directional filters.

\section{REFERENCES}

[1] J. Zi, J. Wan, and C. Zhang, Appl. Phys. Lett. 73, 2084-2086, 1998.

[2] X. Wang, X. Hu, Y. Li, W. Jia, C. Xu, X. Liu, and J. Zi, Appl. Phys. Lett. 80, 4291, 2002.

[3] J. Lekner, J. Opt. A, Pure Appl. Opt. 2, 349, 2000.

[4] E. Yablonovitch, Opt. Lett. 23, 1648, 1998.

[5] J. Xu, H. Fang and Z. Lin, J. Phys. D: Appl. Phys. 34, 445-449, 2001.

[6] H-Y. Lee and T. Yao, J. Appl. Phys. 93, 819830, 2003.

[7] H. Daninthe, S. Foteinopoulou, C. M. Soukoulis, Photonics and NanostructuresFundamentals and Applications 4, 123-131, 2006.

[8] G.N. Pandey, Khem. B. Thapa, and S .P. Ojha, Journal of Ovonic Research (U S A), Vol.6, 87-92, 2010.

[9] G.N. Pandey, K. B. Thapa and S.P. Ojha, Optik - International Journal for Light and Electron Optics, Volume 124, Issue 18, Pages.3396-340, 2013.

[10] G. N. Pandey, J. P. Pandey, A, K, Mishra and S. P. Ojha, Proceedings of the International Conference on "Condensed Matter \& Applied Physics" (ICC- 2015)” 
AIP Conference Proceedings, Volume 1728, pp 020312-1-020312-4, (2016).

[11] G. N. Pandey, J. P. Pandey, U. K. Pandey, Bhagyashree Sancheti and S. P. Ojha, Proceedings of the International Conference on "Condensed Matter \& Applied Physics" (ICC- 2015)” AIP Conference Proceedings, 1728, pp 020306-1-020306-4, (2016).

[12] J P Pandey, International Journal of Pure and Applied Physics, Volume 13, Number 2 pp. 167-173, 2017.

[13] J P Pandey, IOSR Journal of Applied Physics (IOSR-JAP), Volume 9, Issue 2, pp 59-63 Ver. I (Mar. - Apr. 2017).

[14] P. Yeh, "Optical waves in layered media", John Wiley \& sons. U.S.A., 1988.

[15] H. T. Jiang, H. Chen, H. Q. Li. Y. W. Zhang, and S. Y. Zhu, Appl. Phys. Lett. 83. 5386, 2003.

[16] D. R. Smith and N. Kroll, Phys. Rev. Lett.85, 2933, 2000.

[17] R. W. Ziolkowski, Phys. Rev. E 70, 046608, 2004.

[18] G. V. Eleftheriades, A. K. lyer. and P.C. Kremer, IEEE Trans. Microw, Theory Tech. 50, 2702, 2002.

[19] L. W. Zhang. Y. W. Zhang, L. He, H. Q. Li. and H. Chen, Phys: Rev. E 74, 056615, 2006.

[20] Y. J. Xiang, X.Y. Dai, S.C. Wen, and. D. Y. Fan, Phys. Rev. E 76, 056604, 2007. 